\title{
Profile Aerodynamics of an Oscillating Low-Pressure-Turbine Blade
}

\author{
Felix Schwarzbach ${ }^{1,2}$, Dajan Mimic ${ }^{1}$ and Florian Herbst ${ }^{1,2}$ \\ ${ }^{1}$ Institute of Turbomachinery and Fluid Dynamics \\ Leibniz Universität Hannover \\ An der Universität 1, Garbsen 30823, Germany \\ mimic@tfd.uni-hannover.de \\ ${ }^{2}$ Former Member of the Institute of Turbomachinery and Fluid Dynamics \\ Leibniz Universität Hannover
}

\begin{abstract}
This paper presents an analysis of the vibration-induced effects on the aerofoil aerodynamics and boundary-layer development of a low-pressure-turbine blade. Large-eddy simulations of an MTUT161 low-pressure-turbine blade with imposed sinusoidal rigidbody oscillations were conducted for frequencies of 50 and $100 \mathrm{~Hz}$ as well as for a fixed reference blade.

The oscillations are shown to impact both the time-averaged flow field and unsteady velocity fluctuations. These changes appear most markedly as a reduction in the stagnation-point pressure and a partial suppression of the separation bubble on the suction side of the aerofoil. The results suggest that the deterministic velocity fluctuations introduced by the oscillating blade promote transition on the suction side and expedite the generation of turbulence.
\end{abstract}

\begin{tabular}{|c|c|}
\hline $\begin{array}{l}\text { NOM } \\
\text { Symb }\end{array}$ & ATURE \\
\hline$c$ & chord length \\
\hline$c_{p}$ & pressure distribution \\
\hline$f$ & frequency \\
\hline$k$ & reduced frequency \\
\hline$L_{\mathrm{T}}$ & turbulent length scale \\
\hline $\mathrm{Ma}_{\mathrm{th}}$ & theoretical Mach number \\
\hline$n^{+}$ & non-dimensional distance normal to the wall \\
\hline$p$ & pressure \\
\hline$P_{\mathrm{UKE}}$ & production of unsteady kinetic energy \\
\hline $\mathrm{Re}_{\text {th }}$ & theoretical Reynolds number \\
\hline$t$ & time \\
\hline$t^{+}$ & non-dimensional distance tangential to the wall \\
\hline $\mathrm{Tu}$ & turbulence intensity \\
\hline$U_{\text {Mag }}$ & magnitude of velocity \\
\hline$U_{\mathrm{t}}$ & stream-wise/tangential velocity \\
\hline$U_{\infty}$ & free-stream velocity \\
\hline$u_{\mathrm{i}}$ & component of velocity \\
\hline$u_{\mathrm{i}}^{\prime}$ & fluctuation of velocity component \\
\hline UKE & unsteady kinetic energy \\
\hline$h_{\mathrm{n}}$ & wall-normal distance \\
\hline$h_{\text {Pitch }}$ & pitch-wise distance \\
\hline$z^{+}$ & non-dimensional distance in span-wise direction \\
\hline
\end{tabular}

Presented at International Gas Turbine Congress 2019 Tokyo, November 17-22, Tokyo, Japan

Review Completed on, September 17, 2020

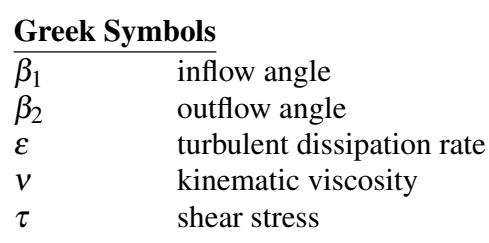

\begin{tabular}{ll}
\multicolumn{2}{l}{ Abbreviations } \\
DFSEM & divergence-free synthetic-eddy method \\
LES & large-eddy simulation \\
LPT & low-pressure turbine \\
PISO & pressure-implicit with splitting of operators \\
RANS & Reynolds-averaged Navier-Stokes \\
SRS & scale-resolving simulations \\
URANS & unsteady Reynolds-averaged Navier-Stokes \\
WALE & wall-adapting local eddy-viscosity
\end{tabular}

\section{INTRODUCTION}

Turbomachine blades vibrate under certain operating conditions. These vibrations can be self-induced, such as flutter, or caused by external excitation, e.g., by moving wakes from upstream blade rows. The accurate and reliable prediction of such blade vibrations resulting from the interaction between fluid and structure is crucial as it allows designers to avoid the often dire consequences of blade flutter, which may result in catastrophic failure of a turbomachine. Beyond this obvious reasoning, surface vibrations have been shown to affect the development of boundary layers and flow separations, e.g., Ref. [1]. As will be apparent from the results and analyses presented in this paper, such alterations of the boundary-layer state bear the potential to impact the instantaneous and time-averaged blade loading and even the flow downstream of the blade.

The transition-like nature of the mechanism driving these alterations is, to the knowledge of the authors, not represented in current turbulence or transition models used for the closure of the Reynolds-averaged Navier-Stokes (RANS) equations. In addition, linear eddy-viscosity turbulence models tend to struggle with the prediction of turbulence near stagnation points: in the case of a vibrating surface, the entire surface may act as an instantaneous stagnation-point flow. 
Revell et al. [2] mentioned that the Boussinesq assumption implies that the strain-rate tensor and resulting Reynolds stress tensor are invariably aligned. This leads to erroneous behaviour of conventional eddy-viscosity turbulence models for unsteady flow where strains and stresses are always in phase. As a result, such models exhibit deficits in predicting the production and dissipation rates of turbulent kinetic energy under cyclic strain [2]. Likewise, the prediction of more turbomachinery-specific flows, i.e., in vibrating cascades, as well as the resulting prediction of aero-elastic quantities, using unsteady RANS (URANS) or time-linearised RANS exhibits non-negligible discrepancies from experimental validation data, e.g., Refs [3-5].

These deficiencies of current RANS models do not only yield inaccurate performance predictions but also preclude further understanding of the physical mechanisms in action. Due to the small scales presumably involved, scale-resolving simulations (SRS) present a viable alternative to approach the problem of vibrationinduced flow phenomena in turbomachinery.

Specifically, large-eddy simulations (LES) are a suitable method to simulate flows at moderate Reynolds numbers with sufficient accuracy and affordable computational cost. Turbulence in turbomachinery can be analysed thoroughly with LES as shown in Refs [6] and [7]. However, the quality of the results depends on the subgridscale model used [8]; a good agreement between LES and DNS was reached by Michelassi et al. who performed LES with periodicaletly impinging wakes for a low-pressure-turbine (LPT) profile [9]. Also, studies have shown that LES is a suitable method to predict transition [8]. Schwarzbach et al. showed that an accurate prediction of turbine-aerofoil aerodynamics, including transition and flow separation, can be achieved by imposing suitable artificial inflow turbulence onto an LES simulation.

In this paper, an approach is presented for the investigation of vibration-induced effects on the aerofoil aerodynamics and boundary-layer development of low-pressure-turbine blades. To achieve this goal, large-eddy simulations of an MTU-T161 LPT profile with imposed sinusoidal rigid-body oscillations are analysed. The oscillations are shown to impact both the time-averaged flow field and the unsteady fluctuations.

\section{SIMULATION SETUP}

Large-eddy simulations of an oscillating MTU-T161 lowpressure-turbine aerofoil were conducted with oscillation frequencies being $f=50 \mathrm{~Hz}$ and $f=100 \mathrm{~Hz}$ in addition to a fixed reference blade $(f=0 \mathrm{~Hz})$. In all cases, the wall-adapting local eddyviscosity (WALE) subgrid-scale model by Nicoud et al. [10] was used to simulate the effect of turbulent scales below the resolution of the computational grid. The computations were conducted with the incompressible pressure-based solver pimpleDyMFoam which is part of the OpenFOAM framework. The solver uses the pressure-implicit with splitting of operators (PISO) algorithm. The incompressible solver used here is suitable for the low Mach numbers investigated, i.e., $\mathrm{Ma}<0.1$. A second-order accuracy spatial discretisation - a combination of a $75 \%$ central difference scheme and $25 \%$ upwind scheme-was chosen. A second-order backward scheme was used for the temporal discretisation.

The artificial turbulent inflow was generated using a divergencefree synthetic-eddy method (DFSEM) boundary condition, which was introduced by Poletto et al. [11] and adapted by MüllerSchindewolffs and Herbst [12] to be used in turbomachinery applications. Using this method, a statistically random distribution of ellipsoidal synthetic eddies is generated in a virtual domain. Their induced velocities are subsequently imposed onto the inlet velocity distribution, whereafter they decay into natural turbulence that is characterised by the time-averaged mean values of the turbulence intensity Tu and turbulent length scale $L_{\mathrm{T}}$. A thorough description of the boundary condition can be found in Ref. [12]. The properties used for the DFSEM are shown in Table 1.
Table 1: DFSEM Properties

\begin{tabular}{llll}
\hline \hline Eddy radius & $\begin{array}{l}\text { Reynolds } \\
\text { Stress } \overline{u_{u}^{\prime} u_{i}^{\prime}}\end{array}$ & $\begin{array}{l}\text { Turbulence } \\
\text { Intensity Tu }\end{array}$ & $\begin{array}{l}\text { Turbulent } \\
\text { length Scale } L_{\mathrm{T}}\end{array}$ \\
\hline $3.25 \cdot 10^{-3} \mathrm{~m}$ & $0.26 \mathrm{~m}^{2} / \mathrm{s}^{2}$ & 0.03 & $2 \cdot 10^{-3} \mathrm{~m}$ \\
\hline \hline
\end{tabular}

Table 2: Mesh Properties

\begin{tabular}{ll}
\hline \hline Parameter & Value \\
\hline$n^{+}$ & Leading edge $<1.5$ \\
& Rest of the blade surface $<1$ \\
$t^{+}$ & Near blade $<50$ \\
$z^{+}$ & Near blade $<60$ \\
Non-orthogonality & Max. 59.8 \\
Celltype & Hexahedral \\
Cells & $14.7 \cdot 10^{6}$ \\
\hline \hline
\end{tabular}

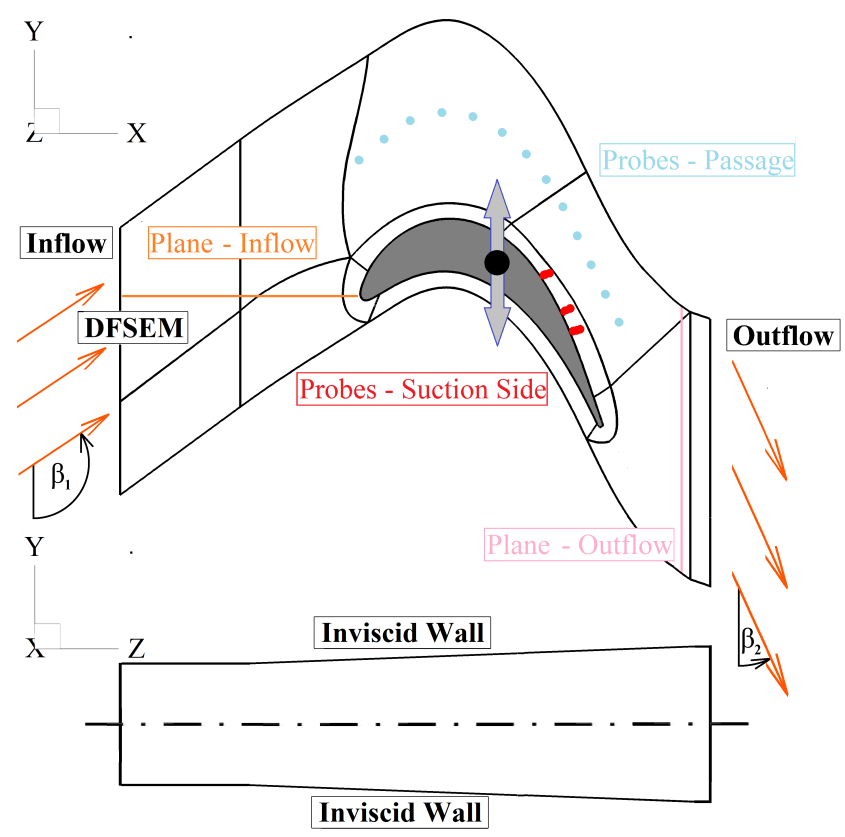

Fig.1: Boundary conditions, probes and direction of the oscillation

The computations were carried out on a mesh with about 1.5 . $10^{7}$ cells. The mesh is described in Refs [13] and [6]. This structured mesh with hexahedral cells was created with ANSYS ICEM CFD by Müller et al. [13] and consists of one O-block and several H-blocks. The mesh properties are shown in Table 2. The domain gives a quasi three-dimensional (Q3D) representation of the MTU-T161 cascade and expands in span-wise direction to satisfy the axial-velocity-density ratio of the original cascade. The walls at both ends of the span are set as inviscid, whereas the pitchwise boundaries are periodic. The time-step size was chosen to be $5 \cdot 10^{-7} \mathrm{~s}$. Using this setup, the maximum subgrid-scale eddy viscosity was significantly lower than the molecular viscosity, reaching peak values of approximately $85 \%(f=100 \mathrm{~Hz})$ and approximately $93 \%(f=50 \mathrm{~Hz})$ of the molecular viscosity in small regions of the wake flow.

The blade oscillations were realised as a rigid-body motion. For each time step, the entire mesh is shifted without any deformation of the cells. This procedure facilitates the use of periodic boundary conditions normal to the blade motion and allows the study of oscillation-induced effects on the profile boundary layer. 
Table 3: Frequencies analysed

\begin{tabular}{llll}
\hline \hline Case & Frequency $f$ & $\begin{array}{l}\text { Reduced } \\
\text { frequency } k\end{array}$ & Amplitude \\
\hline 1 & $0 \mathrm{~Hz}$ & 0 & $0 \mathrm{~m}$ \\
2 & $50 \mathrm{~Hz}$ & 0.45 & $5 \cdot 10^{-3} \mathrm{~m} \hat{=} 8.25 \% c_{\mathrm{Ax}}$ \\
3 & $100 \mathrm{~Hz}$ & 0.9 & $5 \cdot 10^{-3} \mathrm{~m} \hat{=} 8.25 \% c_{\mathrm{Ax}}$ \\
\hline \hline
\end{tabular}

As mentioned at the beginning of this section, two different frequencies, $f=50 \mathrm{~Hz}$ and $f=100 \mathrm{~Hz}$, were chosen (in addition to a fixed reference blade, i.e., $f=0 \mathrm{~Hz}$ ). Defining the chord $c$, frequency $f$ and with a free-stream outlet velocity of $U_{\infty, 2}=21$, as extracted from the fixed reference case, the corresponding reduced frequencies

$$
k=\frac{2 \pi f}{U_{\infty, 2}} \cdot \frac{c}{2} .
$$

can be estimated as $k=0.45$ and $k=0.9$-both typical values encountered in the case of flutter [14]. The parameters used are summarised in Table 3.

Several probes were placed to gather data about the inflow, boundary layers, profile pressure distribution, passage flow and outflow. The probes were defined in the moving blade frame of reference, i.e., they move in unison with the blade. To obtain an accurate representation of the time-averaged flow, $2 \cdot 10^{5}$ instantaneous samples were collected to calculate the temporal average.

\section{REFERENCE CASE AND VALIDATION}

Measurements conducted for the MTU-T161 LPT profile in the low-speed cascade wind tunnel at the Technical University of Braunschweig [15] are used as a reference and validation case for the studies presented in this paper. The wind-tunnel experiments were performed with inlet Mach numbers between 0.046 and 0.23 . The reference case was measured for an inlet Mach number of $\mathrm{Ma}_{\mathrm{th}, 2}=0.046$ and an inflow angle $\beta_{1}=131^{\circ}$. For this case, a Reynolds number of $\mathrm{Re}_{\mathrm{th}, 2}=9 \cdot 10^{4}$ was obtained at the exit plane. The inflow turbulence was generated by means of a turbulence grid consisting of five circular rods with diameters of $0.028 \mathrm{~m}$. The rods were placed $0.125 \mathrm{~m}$ apart in pitch-wise direction. As a result, a turbulence intensity of $2.8 \%$ and a turbulent length scale of $20 \mathrm{~mm}$ were attained at the inlet [15].

An equivalent numerical case, which was presented previously in [6], with a turbulence intensity of $3 \%$ and a turbulent length scale of $20 \mathrm{~mm}$ is used to validate the solver and numerical setup against the experimental data available. Figure 2 shows the blade distributions of the non-dimensional pressure coefficient

$$
c_{p}=\frac{p-p_{2}}{p_{\mathrm{tot}, 1}-p_{2}} .
$$

As can be seen, the numerical simulation gives a nearly perfect prediction of the pressure distribution measured with merely minor differences in the region of the separation bubble: the beginning reattachment of the separation, as indicated by the increase in pressure coefficient at approximately $0.87 c_{\mathrm{Ax}}$, seems to be predicted slightly downstream of its experimental counterpart. However, such small discrepancies may be a due to measurement uncertainties, which are usually exacerbated by the low-speed conditions of the experiment and the resulting small pressure differences across the entire flow field. Figure 3 shows that the prediction of the pitch-wise outflowvelocity distribution is equally satisfying. It follows from the overall accuracy of the predictions that the solver and numerical setup used are suitable and validated sufficiently for the investigations at hand.

While the simulation discussed mimics the experiment precisely, the turbulent length scale of $20 \mathrm{~mm}$ requires a large computational

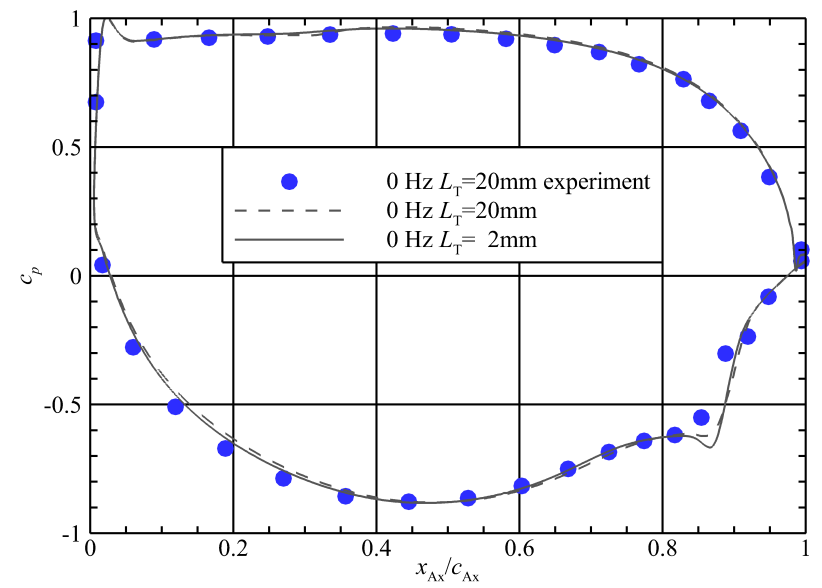

Fig.2: Comparison between simulated and measured pressure distributions along the blade

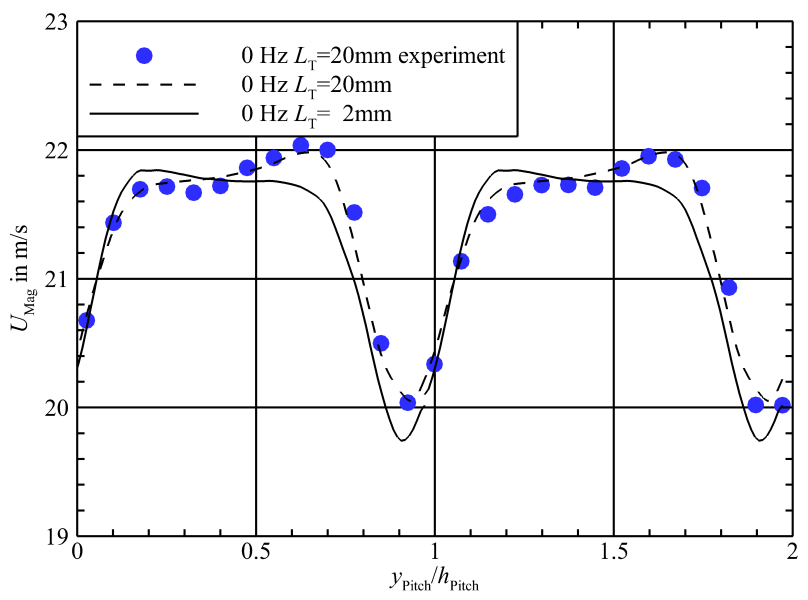

Fig.3: Comparison between simulated and measured pitch-wise outflow-velocity distributions

domain and long sampling duration to obtain a meaningful average. This results in excessively high computational costs for such a simulation to be used in a parameter study. For this reason, a similar setup with a turbulent length scale of $2 \mathrm{~mm}$ at inlet is also shown in Fig. 2. The general picture remains the same: the overall pressure distribution is predicted almost perfectly. Again, differences can be found in the region of the separation bubble on the suction side. Conversely to the higher turbulent length scale, this distribution exhibits a distinct trough at $0.87 c_{\mathrm{Ax}}$. As discussed in [6], this trough is a result of a second, counter-rotating separation just downstream of the initial separation bubble. The outflow-velocity distributions in Fig. 3 show a shift of the peak velocity from the right-hand side to the left-hand side of the plateau and an increase of the wake deficit. The consistency of these results with [6] leads to the conclusion that this setup is physically sound and can be used for the following studies.

\section{RESULTS}

In this section, the effect of a vibrating blade on the surrounding flow field is investigated by imposing pitch-wise, sinusoidal oscillations on the MTU-T161 LPT profile with an inlet turbulent length scale of $2 \mathrm{~mm}$. Frequencies of $50 \mathrm{~Hz}$ and $100 \mathrm{~Hz}$ were chosen for this study.

\section{Pressure Distribution}

The first step to assess the overall impact of blade oscillations on the flow lies in assessing the impact that they have on the blade loading. For this reason, Fig. 4 shows a comparison between the 


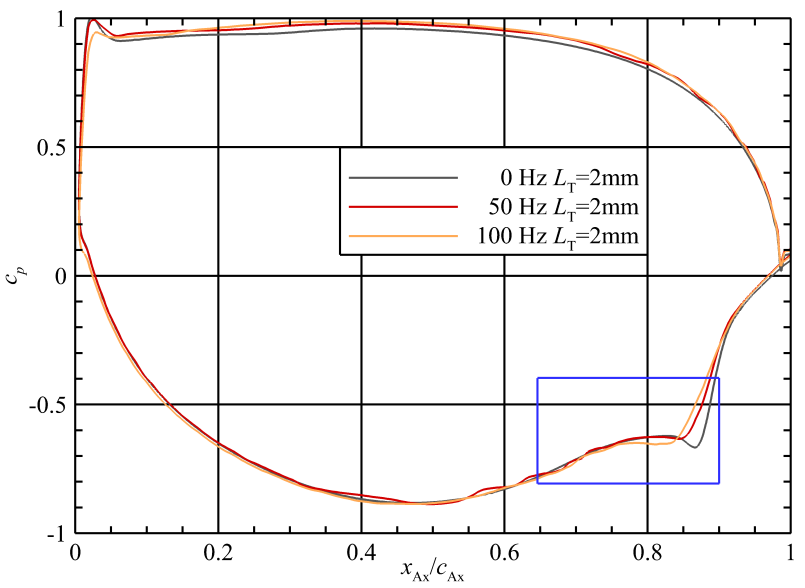

Fig.4: Pressure-coefficient distributions along the blade

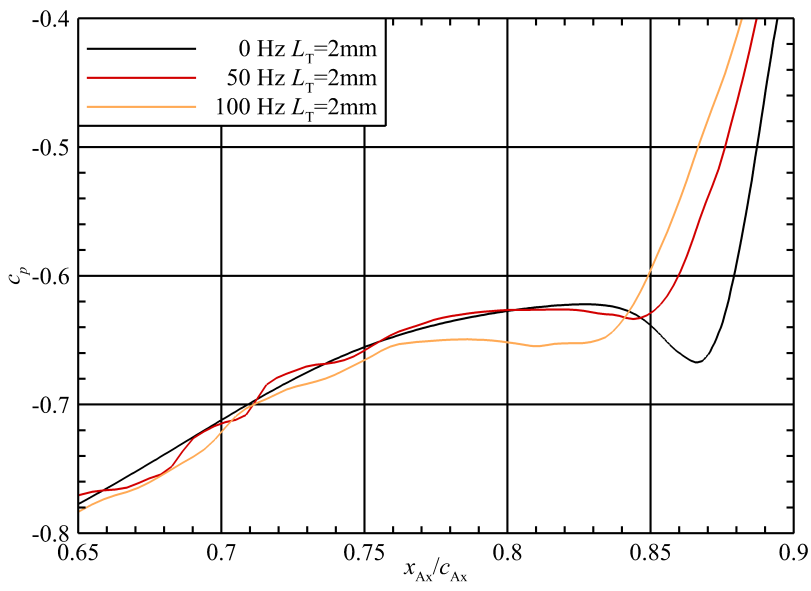

Fig.5: Pressure distribution near the separation bubble

time-averaged blade-pressure-coefficient distributions for the fixed reference case and two oscillating cases. While the figure suggests a similar overall blade loading for increasing frequencies, two major features stand out:

1. The leading-edge stagnation point becomes less prominent and smears out for increasing frequencies.

2. The separation bubble on the suction side seems to be delayed and suppressed partially for increasing frequencies.

The latter point mentioned is shown in detail in Fig. 5, where the separation onset is indicated by a change in curvature of the pressure-coefficient distribution. For the fixed reference $(0 \mathrm{~Hz})$, this point is located at approximately $0.74 c_{\mathrm{Ax}}$; in the case of $50 \mathrm{~Hz}$, it can be estimated at approximately $0.75 c_{\mathrm{Ax}}$ and $0.76 c_{\mathrm{Ax}}$ in the case of $100 \mathrm{~Hz}$. However, the slight wiggling in the pressure-coefficient distributions of the moving blades exhibit just upstream of the timeaveraged separation onset gives rise to the assumption that the separation bubble is actually highly unsteady.

In order to gain an intuitive understanding how the unsteady blade-pressure distribution fluctuates during an oscillation cycle, the pressure coefficients are evaluated instantaneously at four characteristic points of one oscillation period, which are shown in Fig. 6: $0 \pi$, where the blade assumes its zero position with maximum displacement speed towards its suction side; $1 / 2 \pi$, where the blade reaches maximum displacement towards its suction side and zero displacement speed; $1 \pi$, where the blade returns to its zero position with maximum displacement speed towards its pressure side; $3 / 2 \pi$,

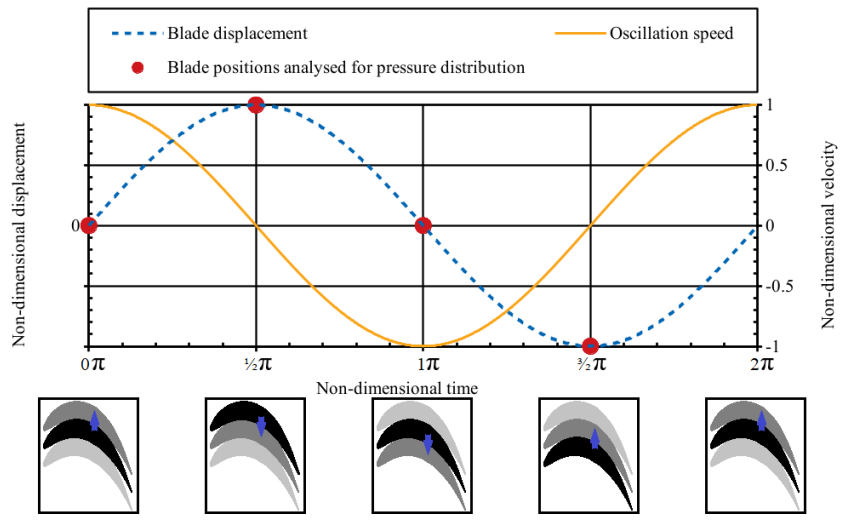

Fig.6: Oscillation period and characteristic positions

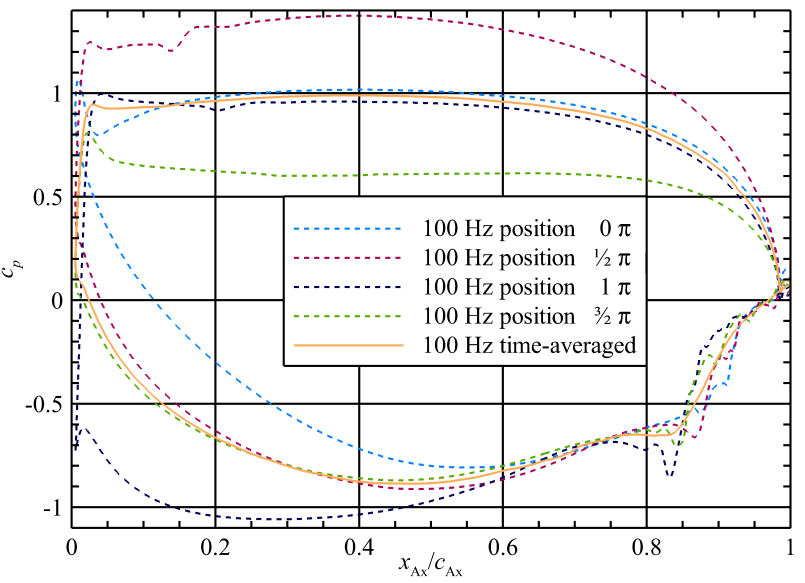

Fig.7: Pressure-coefficient distributions at characteristic positions

where the blade attains maximum displacement towards its pressure side and, again, zero displacement speed.

The resulting instantaneous pressure-coefficient distributions are depicted in Fig. 7 for the example of the $100 \mathrm{~Hz}$ oscillation. The individual graphs show that the blade loading varies significantly over one oscillation period. Likewise, they give evidence of the highly unsteady nature of the stagnation point flow and separation bubble. These variations seem rather unsurprising, considering that the blade movement implies a change in instantaneous incidence. However, a more detailed analysis of the pressure distributions indicates that the change in loading of the pressure side correlates primarily with the displacement of the blade, whereas the stagnation point and suction side seem to be affected strongly by the displacement speed, which is highest at $0 \pi$ and $1 \pi$. The individual flow phenomena found for the flow regions near the stagnation point, pressure side and suction side shall be discussed in the following.

\section{Stagnation-point flow}

The change in the stagnation-point region observed in the timeaveraged pressure-coefficient distribution in Fig. 4 appears as a movement of the stagnation in the time-resolved representation in Fig. 7. The pressure peak is clearly most prominent and furthest upstream at $0 \pi$, where the blade experiences maximum displacement speed towards the suction side, resulting in a strong negative incidence. The immediately following acceleration of the pressureside flow yields to a pressure trough, contributing significantly to a reduction in the time-averaged pressure peak. Likewise, a similar pressure trough can be found further downstream at $1 / 2 \pi$ and $1 \pi$, possibly due to some form of transport. 


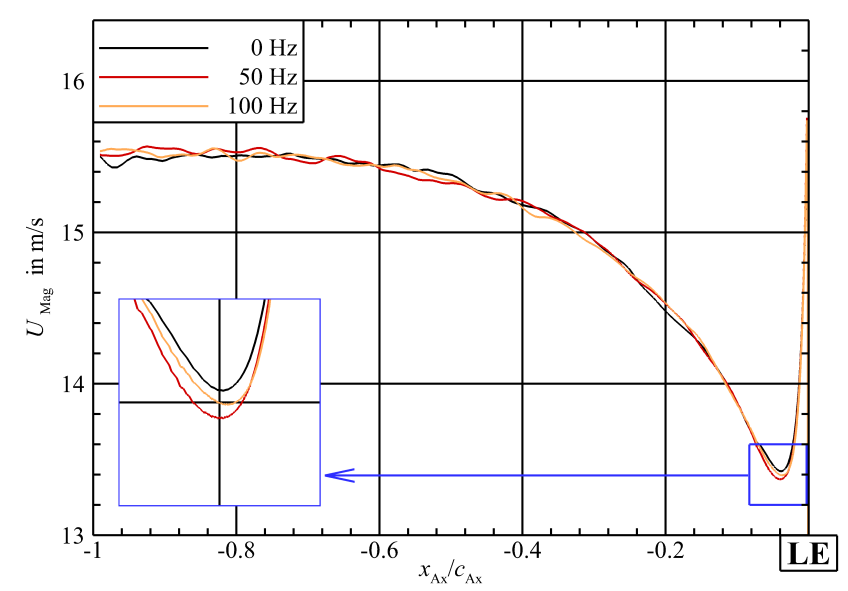

Fig.8: Axial distribution of inflow velocity magnitude $U_{\text {Mag }}$ at the midspan in the frame of reference of the blade

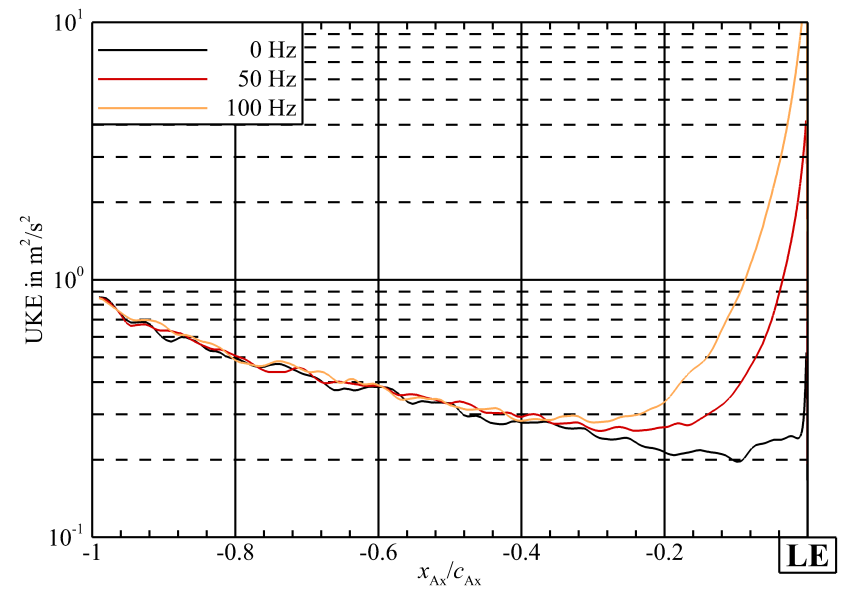

Fig.9: Axial distribution of unsteady kinetic energy (UKE) at the midspan in the frame of reference of the blade

Driven by a positive incidence, the instantaneous pressure peak lies furthest downstream and is the weakest at $1 \pi$. However a suction peak develops just downstream of the leading edge on the pressure side of the blade. A direct comparison of the stagnation-point regions at $1 / 2 \pi$ and $3 / 2 \pi$ reveals that the position of the pressure peak is virtually identical. This is not surprising, as both cases represent terminal blade displacements with displacement velocities of zero. The zero-incidence implied in both cases yields the same location for the stagnation point. However, a difference in the intensity of the peaks (relative to the subsequent plateau) suggests that the incidence is not the sole determining factor. It can be theorised that this temporal asymmetry is caused by the inertia of the surrounding fluid. Certainly, this claim cannot be verified with the data available and requires a more comprehensive analysis of the unsteady flow field, which is beyond the scope of this paper. This contention, however, will be revisited later in this paper in the section Pressureside flow.

To obtain a more comprehensive picture of the flow upstream of the leading edge, the inflow probes (see Fig. 1) shall be considered next. Figure 8 shows the axial distribution of the time-averaged velocity magnitude. All three test cases exhibit a highly similar behaviour, including the strong deceleration towards the leading edge (denoted as 'LE'). However, the resulting trough is slightly deeper for the oscillating blades (see detail in Fig. 8). While the differences are minor, they indicate that the unsteady flow field is impacted by the blade oscillations.

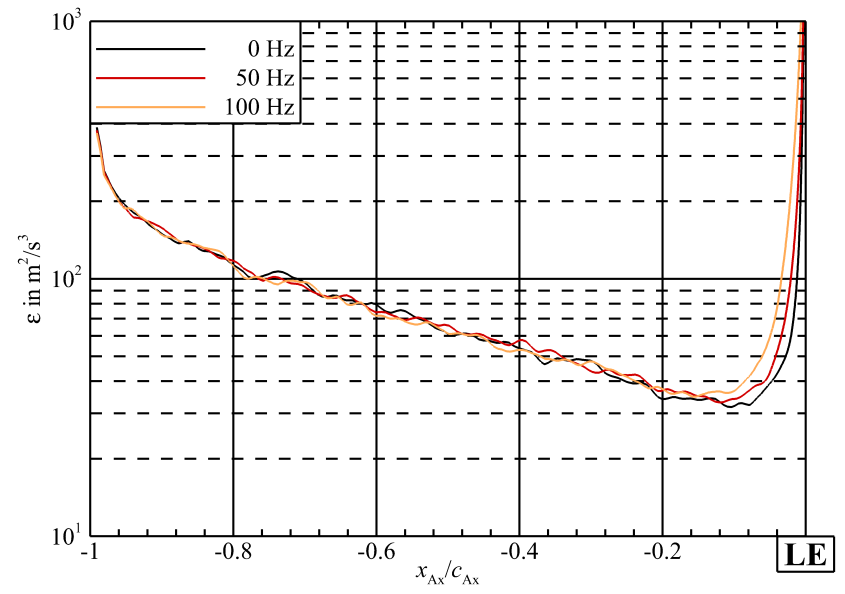

Fig.10: Axial distribution of dissipation rate of UKE epsilon at the midspan in the frame of reference of the blade

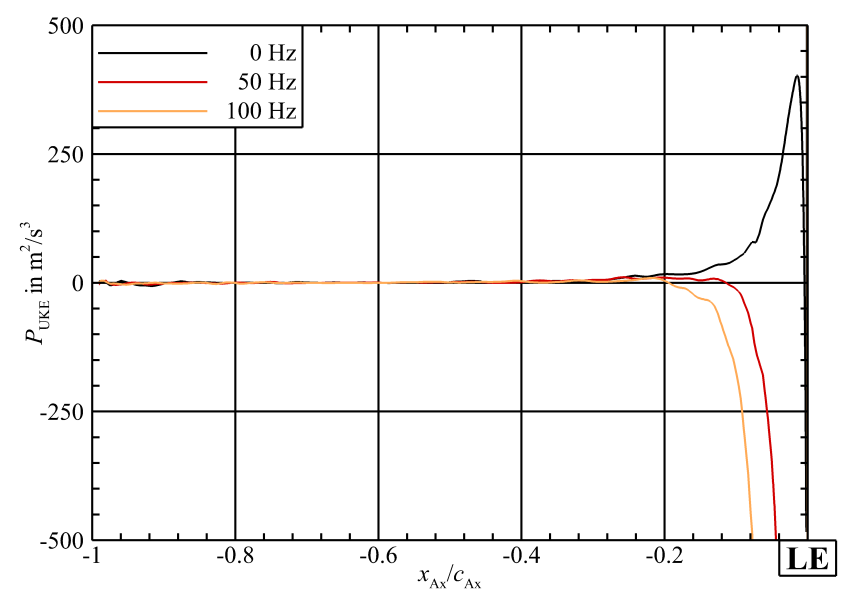

Fig.11: Axial distribution of production of UKE $P_{\mathrm{UKE}}$ at the midspan in the frame of reference of the blade

To delve deeper into the impact on the unsteady flow, the unsteady kinetic energy

$$
\mathrm{UKE}=\frac{1}{2} \overline{u_{i}^{\prime} u_{i}^{\prime}} \text {. }
$$

shall be considered next. Here, the term unsteady kinetic energy is chosen intentionally instead of turbulent kinetic energy, as the contribution of the deterministic fluctuations caused by the relative motion between blade and surrounding fluid is not filtered from the velocity-fluctuation signal. This is deliberate, as the deterministic contribution can still be considered to act similarly to stochastic turbulence in terms of energy transport. The axial distribution in Fig. 9 shows that the UKE increases strongly with the oscillation frequency of the blade. Since both oscillations, $50 \mathrm{~Hz}$ and $100 \mathrm{~Hz}$, have the same amplitude, a higher frequency yields higher displacement speeds for the blade. This, in turn, results in an increased UKE.

Figure 10 shows that the higher UKE for $50 \mathrm{~Hz}$, and more so $100 \mathrm{~Hz}$, leads to a corresponding increase in the dissipation rate of the UKE (analogous to the turbulence dissipation rate), here in its incompressible form:

$$
\varepsilon=\frac{1}{\rho}\left(\overline{\tau_{i j}} \overline{\frac{\partial u_{i}}{\partial x_{j}}}-\overline{\tau_{i j} \frac{\partial u_{i}}{\partial x_{j}}}\right) .
$$

A view of the axial distribution of the production rate of $U K E$ (analogous to the production rate of the TKE), as defined per

$$
P_{\mathrm{UKE}}=-\frac{1}{2} \overline{u_{i}^{\prime} u_{j}^{\prime}}\left(\frac{\partial \overline{u_{i}}}{\partial x_{j}}\right)
$$

is quite intriguing: upon approaching the leading edge, the production rate increases sharply in the case of $0 \mathrm{~Hz}$; for $50 \mathrm{~Hz}$ and $100 \mathrm{~Hz}$ 


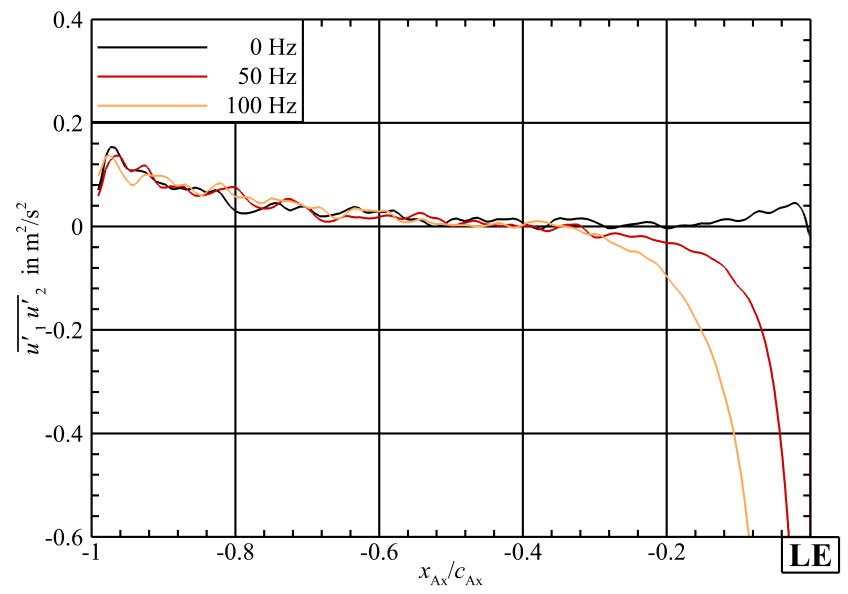

Fig.12: Axial distribution of $u_{1}^{\prime} u_{2}^{\prime}$ correlation at the midspan in the frame of reference of the blade

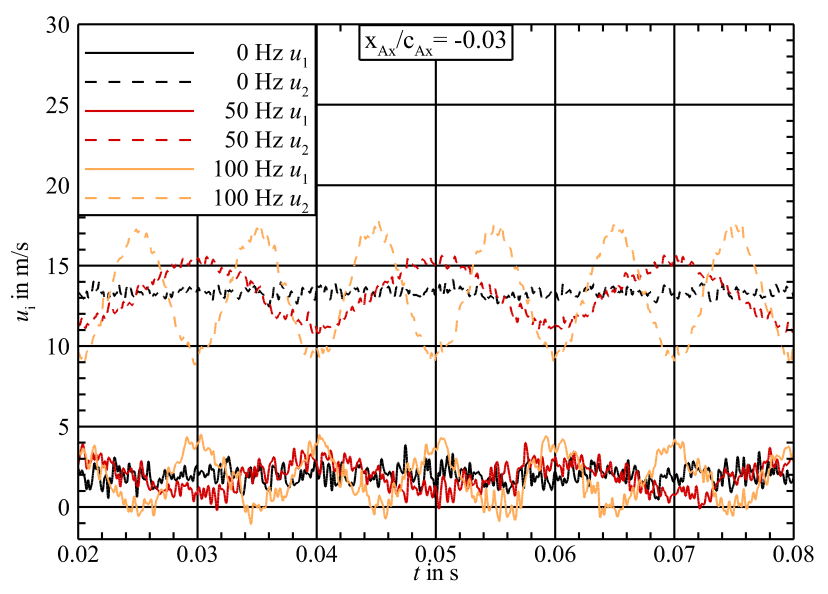

Fig.13: Time-resolved velocity components upstream of the leading edge in the frame of reference of the blade

however, the production rate plummets far below zero. This negative production rate can be understood as an energy transport from the velocity fluctuations back into the mean flow. As concluded in [16], regions of negative production are commonly associated with an increased anisotropy of the turbulence. Figure 12 confirms this notion. The velocity fluctuations exhibit a strong negative $\overline{u_{1}^{\prime} u_{2}^{\prime}}$ correlation close to the oscillating leading edge. The corresponding time-resolved velocity signals just upstream of the leading edge are shown in Fig. 13: a decreased pitch-wise velocity (or negative $u_{2}^{\prime}$ a fluctuation towards the pressure side of the blade) concurs with an increased axial velocity (or positive $u_{1}^{\prime}$ ). This is equivalent to a reduced time-averaged inflow angle. The resulting time-resolved inflow angles $\beta_{1}$ just upstream of the leading edge can be seen in Fig. 14. The phase shift between the oscillations of $u_{1}$ and $u_{2}$ leads to a periodic change of the inflow angle, which, however, is not sinusoidal. An interesting feature of the inflow-angle time series observed is a decrease in the mean value for increasing frequencies (the same decrease as seen via the decreasing $\overline{u_{1}^{\prime} u_{2}^{\prime}}$ correlation in Fig. 12): the time-averaged inflow angle decreases by $0.1^{\circ}$ for $50 \mathrm{~Hz}$ and by $1.1^{\circ}$ for $100 \mathrm{~Hz}$. The result is a slight reduction in the time-averaged blade loading and a greater reduction in the timeaveraged stagnation-point flow.

\section{Pressure-side flow}

As mentioned earlier, the instantaneous pressure level on the pressure side appears to correlate with the displacement of the blade. To be more precise: when the blade is displaced maximally

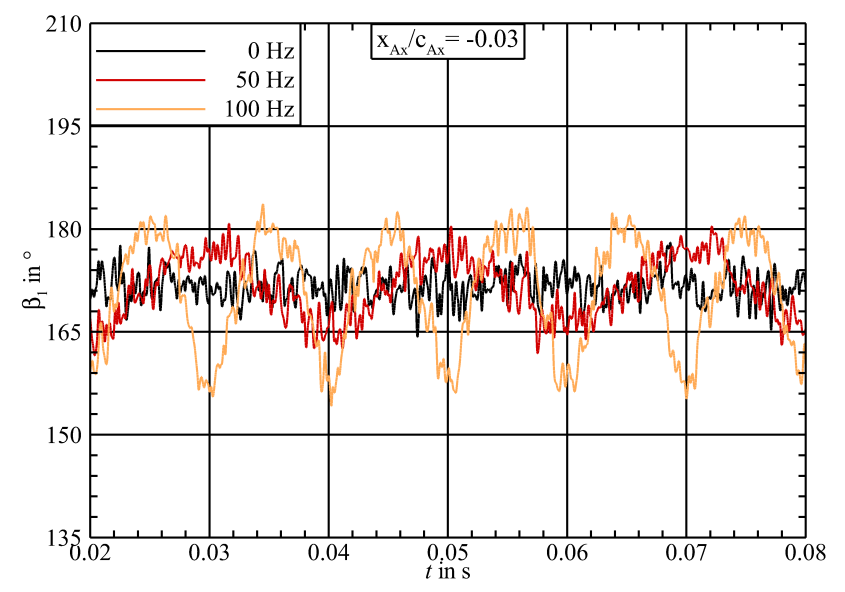

Fig.14: Time-resolved inflow angle upstream of the leading edge at the midspan in the frame of reference of the blade

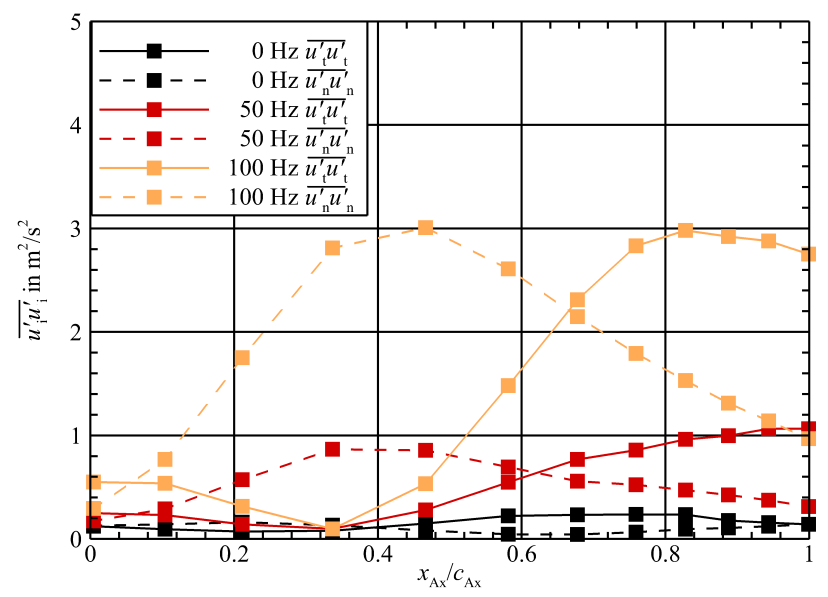

Fig.15: Components of UKE in the passage at the midspan in the frame of reference of the blade

towards its suction side, i.e., at $1 / 2 \pi$, the pressure level is maximal, as well; when the blade is displaced maximally towards its pressure side,i.e., at $3 / 2 \pi$, the pressure level is minimal. This may seem counter-intuitive. However, the displacement speed of the blade decreases as its approaches $1 / 2 \pi$. And as the movement of displacement of the blade decelerates, the inertial force of the fluid moving acts upon the pressure side of the blade. The opposite happens when the blade reaches $3 / 2 \pi$, i.e., maximal displacement towards the pressure side: the movement decelerates and the inertial forces of the fluid act away from the pressure side, leading to a reduced pressure level.

It follows that the pressure-side loading of the blade does not actually depend upon the displacement itself, but rather on its second derivative: the acceleration of the blade movement. Since the displacement follows a sinusoidal form, its acceleration exhibits the same frequency and phase angle. This interpretation is supported by Fig. 15, which shows the time-averaged UKE components tangential and normal to a representative streamline-oriented series of probes in the passage: the velocity fluctuations normal to the probe series (and, therefore, roughly normal to the blade surfaces) are larger for higher oscillation frequencies, and reach their maximum around $0.5 c_{\mathrm{Ax}}$, i.e., where the biggest change in pressure-side loading can be observed.

The explanation for the behaviour of the stagnation-point region at $1 / 2 \pi$ and $3 / 2 \pi$, as mentioned in the section Stagnation-point flow, is likely similar. The different pressure peak intensities observed for both distributions cannot be explained via incidence, but rather by 

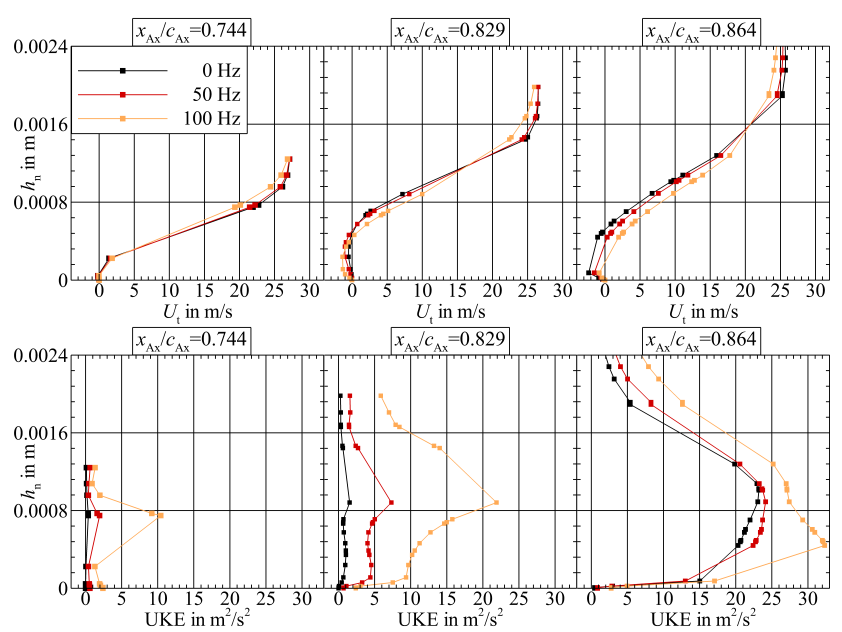

Fig.16: Profiles of streamwise velocity and UKE along the suction side close to the separation-induced transition
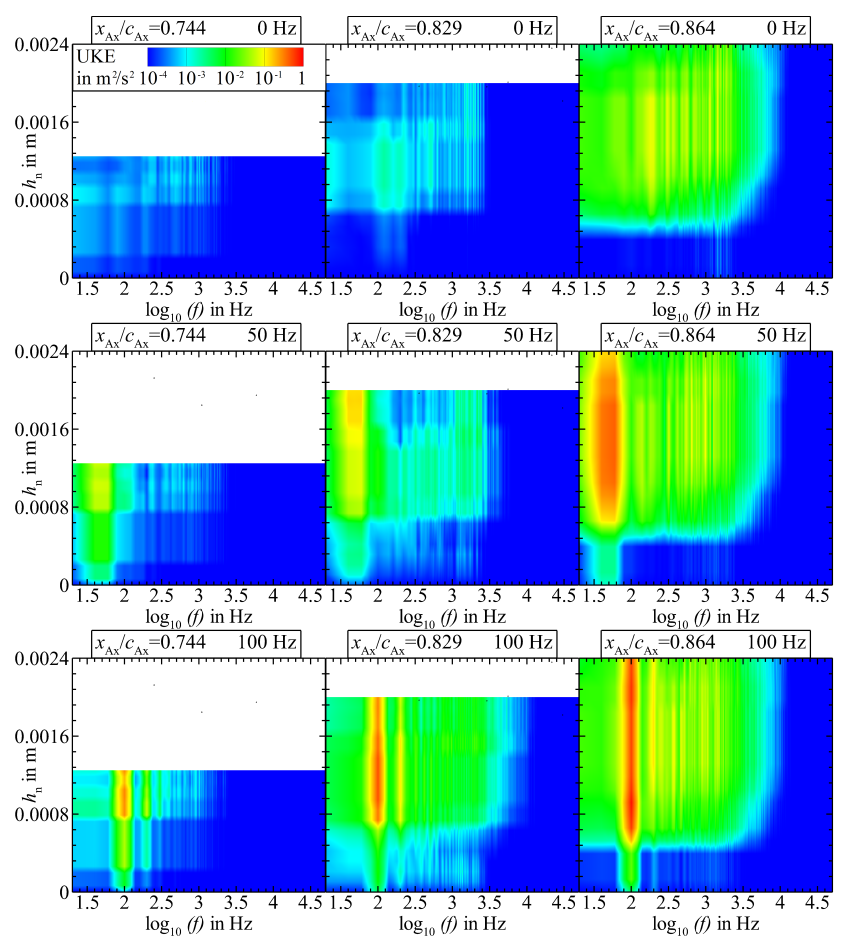

Fig.17: UKE spectra along the suction side close to the separationinduced transition

the inertia of the fluid. The effect at the stagnation point, however, is less pronounced. It can be argued that the fluid in this flow region partially runs off of the convex shape of the leading edge, wherefore it is neither accelerated nor decelerated as effectively and inertial forces play a less important role. The concave form of the pressure side, in turn, facilitates high inertial forces.

\section{Suction-side flow}

In contrast to the pressure-side loading, which correlates with the second derivative of the displacement, the suction-side loading seems to depend upon the first derivative of the displacement, i.e., its speed. Here, the loading is minimal at $0 \pi$ of the oscillation period and maximal at $1 \pi$, as shown in Fig. 7 . In addition, the separation bubble is more pronounced and moves further upstream at $1 \pi$, but is delayed and partially suppressed at $0 \pi$. One may ask why pressure side and suction side behave so differently. It can be inferred that the movement of the blade leads to a displacement of the adjacent fluid. It seems reasonable to suppose that the fluid is not only displaced pitch-wise but also deflected along the convex sur-

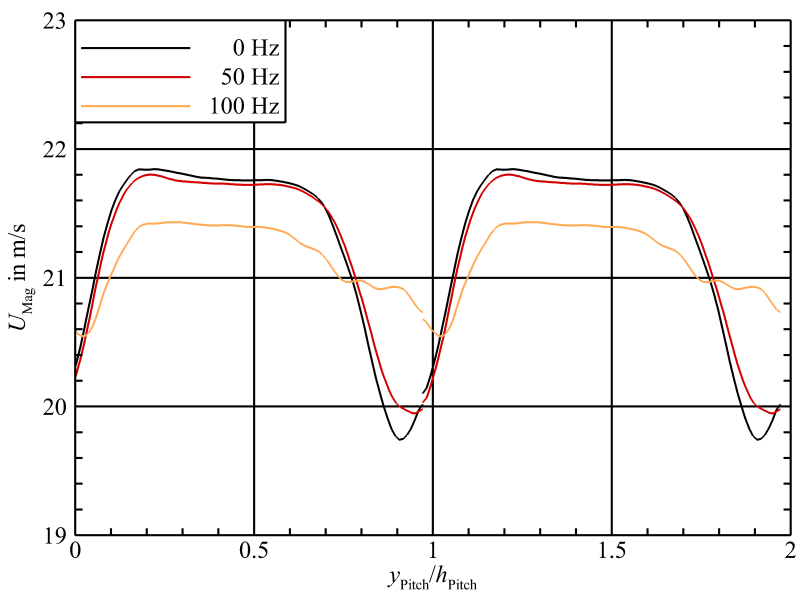

Fig.18: Velocity distribution along the outflow plane at midspan position in the frame of reference of the blade

face of the suction side. Therefore, a movement of the blade towards its suction side leads to a reduced flow velocity - and, therefore, ro reduced loading, i.e., increased pressure-along the upstream half of the suction side, and an increased flow velocity along the downstream half of the suction side, which suppresses the separation bubble. The opposite can be assumed when the blade moves towards its pressure side. Figure 15 supports this interpretation: the largest velocity fluctuations tangential to the probe series (and, therefore, roughly tangential to the blade surfaces) can be observed in the upstream and downstream part of the passage, whereas they vanish in the mid-section.

The time-averaged separation-suppressing effect of the oscillation can also be seen in the upper row of Fig. 16, where the bladenormal profiles of the streamwise, or tangential, velocity are shown. The bottom row of Fig. 16 shows the corresponding UKE profiles of the boundary layer. The blade vibrations result in a clearly increased level of UKE. A spectral analysis, depicted in Fig. 17 evidences that the UKE is mostly concentrated in a narrow frequency band around the oscillation frequency $(50 \mathrm{~Hz}$ and $100 \mathrm{~Hz})$. Close to the onset of the separation-induced transition (from $0.744 c_{\mathrm{Ax}}$ to $0.829 c_{\mathrm{Ax}}$ ), the UKE breaks down into several harmonic bands and eventually into a broadband distribution. It seems that the turbulence produced during the separation-induced transition process feeds on the kinetic energy stored in the deterministic fluctuations. As a result the transition is amplified in the case of an oscillating blade. At $0.864 c_{\mathrm{Ax}}$, however, the broadband UKE is slightly lower overall for $50 \mathrm{~Hz}$ than for $0 \mathrm{~Hz}$, while it remains higher for $100 \mathrm{~Hz}$. It can, therefore, be inferred that different frequencies impact the balance between production and dissipation mechanisms to a different extent.

\section{Outflow}

Turbomachines generally comprise multiple blade rows and stages. It follows that the impact of blade vibrations on the outflow is highly important, since it represents the inflow for the subsequent blade row.

To investigate the effect that the oscillation has on the outflow, Fig. 18 shows the time-averaged pitch-wise distribution (all probes are in the moving frame of reference of the blade) of the velocity magnitude for all three cases studied. The suppression of the separation bubble leads to a reduction in the wake deficit for the vibrating blades; this is especially pronounced for $100 \mathrm{~Hz}$, where the wake deficit is almost halved. The resulting reduction in blockage and momentum loss then leads to a lower free-stream velocity, as the simulations were performed with constant time-averaged inlet velocity and outlet pressure. This is accompanied by overall higher levels of UKE and a slightly more axially oriented outflow for $50 \mathrm{~Hz}$ and, even more so, for $100 \mathrm{~Hz}$, which is not shown here. 


\section{CONCLUSIONS}

In this paper, an approach was presented to investigate vibrationinduced effects on the aerofoil aerodynamics and boundary-layer development of low-pressure-turbine blades. To this end, largeeddy simulations of an MTU T161 LPT profile with imposed sinusoidal rigid-body oscillations were performed, giving accurate results. The oscillations were shown to have a sweeping impact on the flow, which can be concluded as follows:

1. The oscillations show a time-averaged effect on the blade loading, stagnation-point flow and separation bubble.

2. The blade oscillations lead to a region of negative production of unsteady kinetic energy (unfiltered turbulent kinetic energy, which contains the excitation frequency) which is linked to an increased anisotropy of the velocity fluctuations. This appears as a reduction in the time-averaged inflow angle when the blade is oscillating, i.e., a more axially oriented inflow.

3. The unsteady kinetic-energy fluctuations induced by the blade oscillation reduce the separation bubble on the suction side significantly.

4. The oscillations affect the separation-induced transition process on the suction side. The large-scale fluctuations caused by the oscillation seem to feed the turbulence generated. Slightly downstream of the transition point, however, the broadband turbulence is reduced for the $50 \mathrm{~Hz}$ oscillation, while it remains increased for the $100 \mathrm{~Hz}$ oscillation. This suggests a shift in the balance between production and dissipation mechanisms, depending upon the oscillation frequency.

5. The pitch-wise distribution of the outflow velocity is more homogeneous for higher oscillation frequencies. This is likely a consequence of the suppressed separation bubble.

The next step needs to be a comparison of common RANS and URANS models to the data presented here, in order to determine the exact prediction deficits with regards to turbomachinery-specific flows and in which way these deficits affect the prediction of aerodynamic damping and, therefore, the prediction of flutter. Based upon the results of this next step, RANS-modeling approaches will have to be derived. This will likely require a thorough analysis of the contribution of production and dissipation rates to the various scales of turbulence or turbulent-like fluctuations encountered.

\section{ACKNOWLEDGEMENTS}

The scale-resolving simulations have been completed with resources of the North-German Supercomputing Alliance (HLRN) from the HPC Gottfried in Hannover, Germany. The project is part of the research alliance MOBILISE, which is a cooperation between Leibniz Universität Hannover and Technische Universität Braunschweig. The authors would like to thank MTU for providing the data for the MTU-T161 LPT cascade. The experimental measurements were provided by the Institute of Jet propulsion and Turbomachinery (IFAS) at the Technische Universität Braunschweig. The authors would further like to thank Mark Zieße, who contributed to the post-processing routines used in this paper.

\section{References}

[1] Sutton, E.P., Evans, G. P., McGuinness, M.D., and Svehla, K.M., 1981, "Influence of Wall Vibrations on a Flow With Boundary-Layer Separation at a Convex Edge". In Unsteady Turbulent Shear Flows, International Union of Theoretical and Applied Mechanics. doi: 10.1007/978-3-642-81732-8_23

[2] Revell, A.J., Benhamadouche, S., Craft, T., and Laurence, D., 2006, "A stress-strain lag Eddy viscosity model for unsteady mean flow". International Journal of Heat and Fluid Flow, Vol.27(5), pp. 821-830, doi: 10.1016/j.ijheatfluidflow.2006.03.027
[3] Kersken, H., Frey, C., Voigt, C., and Ashcroft, G., 2011, "Time-Linearized and Time-Accurate 3D RANS Methods for Aeroelastic Analysis in Turbomachinery". ASME J. Turbomach., Vol.134(5), p. 8, doi: 10.1115/1.4004749

[4] Purushothaman, K., Jeyaraman, S.K., Parida, S., and Deshkulkarni, K.P., 2017, "Aeroelastic Flutter Analysis of Linear Cascade Blades: STC5". In Proc. ASME GT India, Vol.1, p. 8. doi: 10.1115/GTINDIA2017-4773

[5] Slama, V., Rudas, B., Ira, J., Macalka, A., Eret, P., and Tsymbalyuk, V., 2018, "The Validation of Flutter Prediction in a Linear Cascade of Non-Rigid Turbine Blades". In Proc. ASME Turbo Expo, Vol.7C, p. 10. doi: 10.1115/GT201875502

[6] Schwarzbach, F., Müller-Schindewolffs, C., Bode, C., and Herbst, F., 2018, "The Effect of Turbulent Scales on LowPressure Turbine Aerodynamics: Part B - Scale Resolving Simulations". In Proc. ASME Turbo Expo, Vol.2B, p. 13. doi: 10.1115/GT2018-75163

[7] Pichler, R., Zhao, Y., Sandberg, R.D., Michelassi., V., Pacciani, R., Marconcini, M., and Arnone, A., 2018, "LES and RANS Analysis of the End-Wall Flow in a Linear Cascade, Part I: Flow and Secondary Vorticity Fields under Varying Inlet Condition". In Proc. ASME Turbo Expo, Vol.2B, p. 11. doi: 10.1115/GT2018-76450

[8] Di Pasquale, D., and Rona, A., and Garrett, S.J., 2009, “A Selective Review of CFD Transition Models". In Proceedings of 39th AIAA Fluid Dynamics Conference, San Antonio, Texas, p. 10. doi: $10.2514 / 6.2009-3812$

[9] Michelassi, V., Wissink, J.G., Fröhlich, J., and Rodi, W., 2003, "Large Eddy Simulation of a Flow around a Turbine Blade with Incoming Wakes". AIAA Journal, Vol. 41(11), pp. 2143 2156. doi: $10.2514 / 2.6832$

[10] Nicoud, F., and Ducros, F., 1999, "Subgrid-scale stress modelling based on the square of the velocity gradient". Flow, Turb. and Combustion, Vol.62(3), pp. 183-200. doi: 10.1023/A:1009995426001

[11] Poletto, R., Craft, T., and Revell, A.J., 2013, “A New Divergence Free Synthetic Eddy Method for the Reproduction of Inlet Flow Conditions for LES". Flow Turbulence Combust, Vol.91(3), pp. 519-539. doi: 10.1007/s10494-013-9488-2

[12] Müller-Schindewolffs, C., and Herbst, F., 2018, "The Effect of Turbulent Scales on Low-Pressure Turbine Aerodynamics - Part A: An optimized Turbulent BoundaryCondition". In Proc. ASME Turbo Expo, Vol.2B, p. 16. doi: 10.1115/GT2018-75162

[13] Müller, C., Baier, R.-D., Seume, J.R., and Herbst, F., 2016, "DNS-Based Analysis of RANS Predictions of a LowPressure Turbine Cascade”. J. Turbomach., Vol.139(8), pp. 081006-1-081006-11. doi: 10.1115/1.4035834

[14] Fleeter, S., and Jay, R.L., 1987, "Unsteady Aerodynamic Measurements in Flutter Research". In Unsteady Turbomachinery Aerodynamics, pp. 3-42, AGARD-AG298.

[15] Bode, C., Aufderheide, T., Kožulović, D., and Friedrichs, J., 2014, "The Effects of Turbulence Length Scale on Turbulence and Transition Prediction in Turbomachinery Flows". In Proc. ASME Turbo Expo, Vol.2B, p. 13. doi: 10.1115/GT201427026

[16] Liberzon, A., Lüthi, B., Guala, M., Kinzelbach, W., and Tsinober, A., 2005, "Experimental study of the structure of flow regions with negative turbulent kinetic energy production in confined three-dimensional shear flows with and without buoyancy". Physics of Fluids, Vol.17(9). doi: $10.1063 / 1.2055447$ 